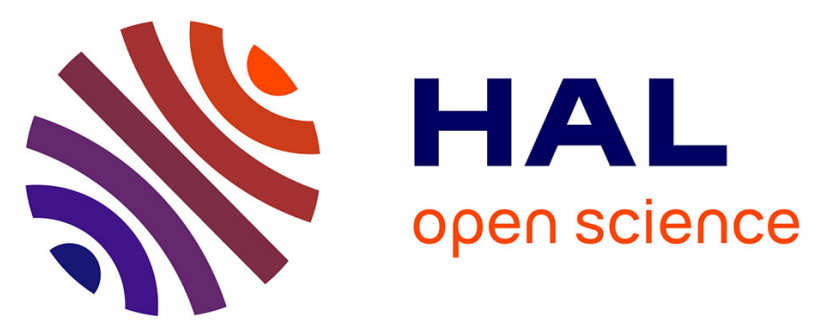

\title{
Analysis of electroluminescence spectra of silicon and gallium arsenide $p-n$ junction in avalanche breakdown
}

\author{
M. Lahbabi, A. Ahaitouf, M. Fliyou, E. Abarkan, J.P. Charles, A. Bath, A.
} Hoffmann, S.E. Kerns, D. V. Kerns Jr.

\section{- To cite this version:}

M. Lahbabi, A. Ahaitouf, M. Fliyou, E. Abarkan, J.P. Charles, et al.. Analysis of electroluminescence spectra of silicon and gallium arsenide p-n junction in avalanche breakdown. Journal of Applied Physics, 2004, 95 (4), pp.1822-1828. 10.1063/1.1643188 • hal-00323131

\section{HAL Id: hal-00323131 \\ https://hal.science/hal-00323131}

Submitted on 2 Dec 2021

HAL is a multi-disciplinary open access archive for the deposit and dissemination of scientific research documents, whether they are published or not. The documents may come from teaching and research institutions in France or abroad, or from public or private research centers.
L'archive ouverte pluridisciplinaire HAL, est destinée au dépôt et à la diffusion de documents scientifiques de niveau recherche, publiés ou non, émanant des établissements d'enseignement et de recherche français ou étrangers, des laboratoires publics ou privés. 


\title{
Analysis of electroluminescence spectra of silicon and gallium arsenide $p-n$ junctions in avalanche breakdown
}

\author{
M. Lahbabi and A. Ahaitoufa) \\ Unité de Formation et de Recherche, Signaux Systèmes et Composants, Laboratoire L2SC, Faculté des \\ Sciences et Techniques, Boite Postale 2202, Fès, Morocco \\ M. Fliyou \\ Ecole Normale Supérieure, Marrakech, Morocco \\ E. Abarkan \\ Unité de Formation et de Recherche, Signaux Systèmes et Composants, Laboratoire L2SC, Faculté des \\ Sciences et Techniques, Boite Postale 2202, Fès, Morocco \\ J.-P. Charles and A. Bath \\ Materiaux Optique Photonique et Systèmes, (CNRS FRE 2304), Université de Metz, 2 Rue Edouard Belin, \\ 57000 Metz, France
}

\begin{abstract}
A. Hoffmann
Centre d'Electronique et de Micro-opto-électronique de Montpellier (CNRS UMR 5507), CC 084, Université de Montpellier 2, Place Eugène Bataillon, 34095 Montpellier Cedex 5, France
\end{abstract}

S. E. Kerns and D. V. Kerns, Jr. Franklin W. Olin College of Engineering, 1735 Great Plain Avenue, Needham, Massachusetts 02492

(Received 14 April 2003; accepted 2 December 2003)

\begin{abstract}
We present a generalized study of light emission from reverse biased $p-n$ junctions under avalanche breakdown conditions. A model is developed based on direct and indirect interband processes including self-absorption to describe measured electroluminescence spectra. This model was used to analyze experimental data for silicon $(\mathrm{Si})$ and gallium arsenide $p-n$ junctions and can be extended to several types of semiconductors regardless of their band gaps. This model can be used as a noninvasive technique for the determination of the junction depth. It has also been used to explain the observed changes of the $\mathrm{Si} p-n$ junction electroluminescence spectra after fast neutron irradiation. In particular, it is demonstrated that the neutron irradiation affects both the semiconductor and the overlying passivation oxide layer. (C) 2004 American Institute of Physics.
\end{abstract} [DOI: 10.1063/1.1643188]

\section{INTRODUCTION}

Light emission from reverse biased $p-n$ junctions under high electric field occurs in semiconductor (SC) devices irrespective of their direct or indirect band gaps. In fact, under high electric field, impact ionization occurs and results in avalanche breakdown and creates a large hot-carrier population that yields radiative transitions and/or recombines and produce luminescence. At present, this phenomenon can be activated even under normal operating biases due to recent progress in downsizing SC devices. In practice, avalanche breakdown can be used for high speed light-emitting devices, even high speed light amplifiers, and also to analytically investigate hot carrier distributions. ${ }^{1-3}$ Highly energetic carriers are responsible for the on-state device breakdown which limits the microwave power of high electron mobility transistors (HEMTs) ${ }^{4-6}$ and reduces metal-SC field effect transistor (MESFET) performance. ${ }^{7,8}$ Therefore understanding of carrier luminescence mechanisms is informative for both analysis of device degradation ${ }^{9,10}$ and development of the design of power SC components.

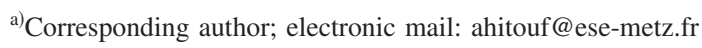

Electroluminescence (EL) spectra of silicon ( $\mathrm{Si}$ ) $p-n$ junctions in avalanche breakdown have been studied for many years ${ }^{11-16}$ but, since Si technology has changed considerably over this period, the purity of microelectronics materials in particular has increased dramatically, and more studies are needed to help clarify overall understanding of the phenomenon. For the most part, recent spectra ${ }^{14-18}$ of $\mathrm{Si}$ $p-n$ junctions have shown a wide hump in the $1.55-3 \mathrm{eV}$ energy range with, most of the time, an emission peak in the visible range at $\sim 2 \mathrm{eV}(620 \mathrm{~nm})$.

The physical mechanisms of photon generation at junctions in avalanche breakdown have been studied and modeled by numerous researchers. For Si $p-n$ junctions, in general, one or more processes among the two following major classes of mechanisms have been used to describe EL spectra:

(1) intraband transitions of electrons or holes within their respective bands; ${ }^{11-13,16-21}$

(2) interband recombination (direct or indirect) of energetic electrons in the conduction band with holes in the valence band. ${ }^{11,13,15,16,22-25}$

In a recent work, ${ }^{16}$ a multimechanism model for photon 
generation by silicon junctions in avalanche breakdown was proposed. It was argued there, that the indirect interband transition is the dominant mechanism producing photons with energies between 1.4 and $2 \mathrm{eV}$, that bremsstrahlung radiation dominates at intermediate energies up to $2.3 \mathrm{eV}$, and that direct recombination between energetic electrons and holes near $k=0$ dominates above $2.3 \mathrm{eV}$. This work reports rather a good fit of experimental data from several labs, although there are some discrepancies between measured and calculated spectra at high energies. Furthermore the transition between indirect interband and bremsstrahlung mechanisms at approximately $2 \mathrm{eV}$ is observed but not rigorously justified. In addition, this model assigns direct interband transitions to energies lower than that of the direct $\mathrm{Si}$ band gap $(\sim 3.1 \mathrm{eV})$. The model has also been used ${ }^{26}$ to study the effect of $30 \mathrm{MeV}$ neutron irradiation on Si $p-n$ junction EL spectra. In all these studies, the silicon self-absorption has always been ignored.

All this work shows that the dominant mechanism for light emission remains under unresolved. In fact, we need a general model which can be applied to any SC type irrespective of its direct or indirect band gap nature.

We have previously reported ${ }^{27}$ a simple model based on interband indirect recombination associated with selfabsorption in the bulk to describe EL spectra from both custom and commercially fabricated $p-n$ Si junctions. This model has been extended successfully to fit GaAs junctions spectra for direct interband recombination. ${ }^{28}$ We have also demonstrated that our model can be used as a noninvasive tool for determination of the local junction depth and the oxide layer thickness. ${ }^{27,28}$

This article focuses on generalization of the model to all SC $p-n$ junctions. It is therefore demonstrated that the interband recombination model which has been applied to $\mathrm{Si}$ can be used to fit the indirect recombination emission observed for GaAs, and to the direct dominant recombination previously modeled ${ }^{28}$ as well. Furthermore our model is used to analyze neutron irradiation effects on the EL spectra measured on $\mathrm{Si} p-n$ junctions and we show in particular that neutron irradiation, for the fluence used, affects both the SC and the oxide passivation layer, unlike the previously reported conclusion ${ }^{26}$ that stated that only the oxide layer properties had changed.

\section{EXPERIMENTAL DETAILS}

The experimental spectra used in this study are of two types.

(1) For $\mathrm{Si}$, the EL spectra were obtained both on custom $\mathrm{Si}$ $p-n$ vertical junctions (phosphorus doped, $N_{D}$ $=10^{19} \mathrm{~cm}^{-3}$, into boron-doped $N_{A}=5 \times 10^{15} \mathrm{~cm}^{-3}$; junction depth $\sim 0.8 \mu \mathrm{m}$ ) and on the gate-drain junction of a commercial Si $n$-channel junction field effect transistors (JFETs) (type 2N4416 fabricated by Siliconix). The reverse current intensity values used to achieve avalanche were 20 and $7 \mathrm{~mA}$ for the $p-n$ junction and JFET, respectively. Figure 1 shows a typical room tem-

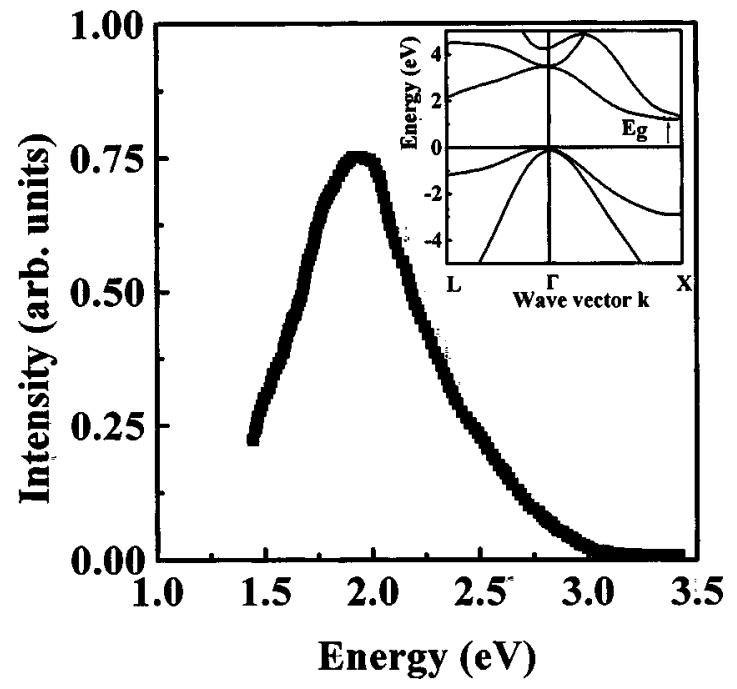

FIG. 1. Typical electroluminescence spectrum measured on the lab custom $p-n$ silicon junction during controlled avalanche breakdown with intensity current $I=20 \mathrm{~mA}$ (after Ref. 16). The Si band structure is shown in the inset.

perature EL spectrum obtained for the custom junction. ${ }^{16}$ This spectrum includes corrections for the spectral efficiency response of the detector.

(2) For GaAs, we have measured EL spectra on two kinds of $p-\mathrm{n}$ junction: (1) $p-n$ junctions supplied by the Centre d'Electronique et de Micro-optoelectronique de Montpellier (CEM2) Laboratory (Montpellier II University) fabricated especially for this study with a junction depth of $1 \mu \mathrm{m}$ and (2) commercially manufactured lightemitting diodes (Siemens IR LD 242).

All the spectra presented were recorded by a microRaman setup, LABRAM 010.

Figures 2 and 3 show representative, uncorrected avalanche breakdown EL spectra amplitude versus the photon energy emitted of custom and commercial junctions, respectively. These measurements were performed at $300 \mathrm{~K}$. The spectra show two peaks. The stronger one (near $1.4 \mathrm{eV}$ ) is

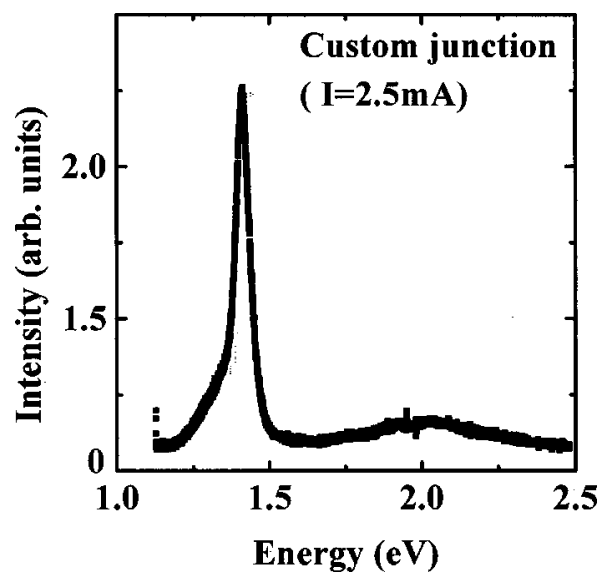

FIG. 2. Measured electroluminescence spectrum of the custom-fabricated GaAs junction during controlled avalanche breakdown $(I=2.5 \mathrm{~mA})$. 


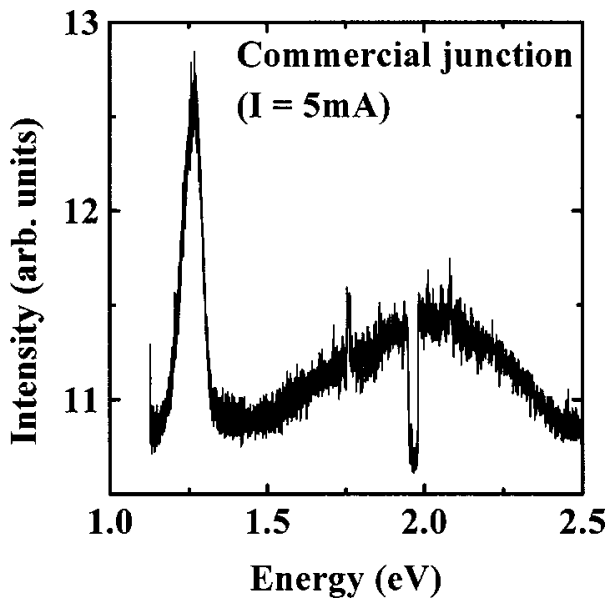

FIG. 3. Measured electroluminescence spectrum of the Siemens IR LD 242 GaAs light-emitting diode during controlled avalanche breakdown ( $I$ $=5 \mathrm{~mA}$ ). The notch near $2 \mathrm{eV}$ is an artifact of the measurement apparatus (red filter suppression).

current dependent. The other peak near $1.9 \mathrm{eV}$ is small and practically independent of the value of current applied.

\section{MODEL}

\section{A. Indirect band gap SC: Silicon case}

Experimental data presented in Fig. 1 show that light emission corresponds to photon energies ranging from 1.4 to $3.4 \mathrm{eV}$ with a pronounced decrease in intensity above $3 \mathrm{eV}$. These energies are larger than that of the indirect Si band gap $(\sim 1.12 \mathrm{eV})$ and localized below the direct $\mathrm{Si}$ band gap $(\sim 3.1$ $\mathrm{eV})$. All possible light emission mechanisms can be analyzed and discussed by placing the experimental spectra (Fig. 1) and the Si band structure ${ }^{29}$ opposite each other. Whenever it occurs, the direct transition from the conduction band to the valence band gives energies above $3 \mathrm{eV}$. Measured EL intensity in this energy range is practically quenched (see Fig. 1), therefore this mechanism cannot be considered here for modeling. The same conclusion is valid for photons generated by the intraband transition of holes in the valence band, which corresponds to energies lower than that of the band gap of $\mathrm{Si}$ (Ref. 20) (infrared radiation) whereas (see Fig. 1) we experimentally measure energies higher than $1.4 \mathrm{eV}$ only. With regard to the intraband transitions of energetic electrons in the conduction band, which correspond to bremsstrahlung radiation, calculations (not reported here) and previous research $^{25}$ show that this radiation exhibits monotonic variation for all energies under the impact ionization threshold energy (2.3 eV for $\mathrm{Si}),{ }^{30}$ whereas experimentally the EL intensity reaches a maximum around $1.9 \mathrm{eV}$ (see Fig. 1). Consequently, this mechanism cannot explain the behavior of the EL spectra in this region or, if it is activated, one must consider that it is dominated by other emission mechanisms. The only other possible process is that of indirect (phononassisted) interband recombination.

According to Yamada and Kiato, ${ }^{25}$ when the phonon energy is neglected compared with the emitted photon energy, the emission rate of photons in the presence of an electric field is expressed as

$$
\begin{aligned}
R_{i}(\hbar \omega) \propto & \int_{0}^{\hbar \omega-E_{g i}} \hbar \omega E^{1 / 2}\left(\hbar \omega-E_{g i}-E\right)^{1 / 2} f(E) \\
& \times[1-f(E-\hbar \omega)] d E,
\end{aligned}
$$

where subscript $i$ refers to indirect, $E_{g i}$ is the indirect band gap, $E$ is the electron energy measured from the conduction band edge, and $f(E)$ is the hot-carrier distribution obtained as a solution of the Boltzmann equation approximated by Wolff $^{22}$ as

$$
f(E)=\exp \left(-\frac{E}{k_{B} T_{e}}\right)\left(1-\frac{\mathbf{E}_{\mathbf{i}}\left(E / k_{B} T_{e}\right)}{\mathbf{E}_{\mathbf{i}}\left(E_{0} / k_{B} T_{e}\right)}\right)
$$

where $E_{0}$ is the threshold energy for impact ionization, $\mathbf{E}_{\mathbf{i}}(x)=\int_{-\infty}^{x} \exp u / u d u, T_{e}$ is the hot-carrier temperature and $k_{B}$ is the Boltzmann constant. $[1-f(E-\hbar \omega)]$ is the hole distribution, taken to be quasi-Maxwellian, that is,

$$
[1-f(E-\hbar \omega)] \approx \exp \left(-\frac{E-\hbar \omega}{k_{B} T_{e}}\right) .
$$

\section{B. Direct band gap SC: GaAs case}

In this case, the emission rate in the presence of an electrical field is given by ${ }^{25}$

$$
R_{d}(\hbar \omega) \propto \hbar \omega\left(\hbar \omega-E_{g d}\right)^{1 / 2} f(E)[1-f(E-\hbar \omega)],
$$

where subscript $d$ refers to direct and $E_{g d}$ is the direct band gap.

\section{Self-absorption effect}

The above modeled emitted photon rate, for direct and indirect recombination processes [Eqs. (1) and (4)], must then be corrected to account for self-absorption, especially for high energy ranges where the absorption coefficients become important for both $\mathrm{Si}$ and GaAs.

The final predicted rate of photon emission is then

$$
R_{s}=R(\hbar \omega) \exp \left[-\alpha(\hbar \omega) x_{j}\right],
$$

where $x_{j}$ is the junction depth, $\alpha(\hbar \omega)$ is the absorption coefficient for a given wavelength, and $R$ denotes either $R_{d}$ or $R_{i}$ given by Eqs. (1) and (4). Note that, in the emission rate expressions [Eqs. (1), (4), and (5)], the hot-carrier temperature $T_{e}$ and junction depth $x_{j}$ are two adjustable parameters. Moreover, $x_{j}$ is a technological parameter which is not always well defined.

\section{RESULTS AND DISCUSSION}

\section{A. Effect of $x_{j}$}

Figures 4 and 5 show computer simulation results versus the junction depth for direct and indirect recombination processes, respectively. The values of $\alpha(\hbar \omega)$ that correspond to GaAs (Ref. 31) are used here because GaAs exhibits two possible transitions. The same analysis can be used for $\mathrm{Si}$ junctions as for the GaAs indirect transition. This is achieved by changing the values of $\alpha(\hbar \omega)$ and $E_{g}$ by using their counterpart Si values. Figures 4 and 5 clearly show that an increase of junction depth reduces the light intensity emitted, especially at higher energies, and shifts the emission peak 


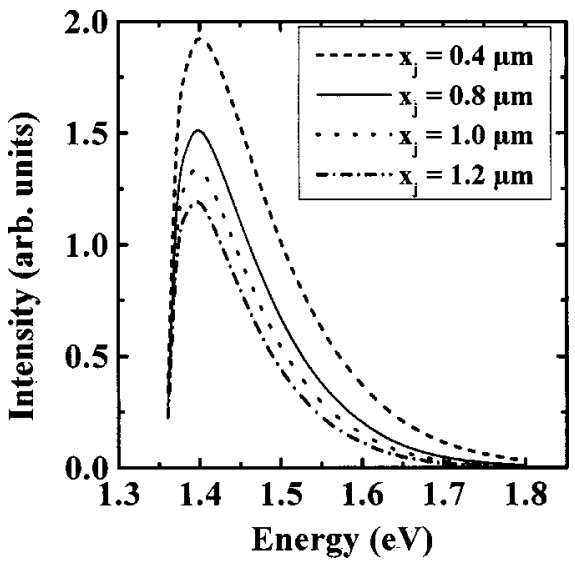

FIG. 4. Simulation of the direct recombination peak intensity for different junctions depths for GaAs.

position towards lower energies. It can be concluded (by comparing Figs. 4 and 5) that the effect of $x_{j}$ is much more pronounced at higher energies where $\alpha(\hbar \omega)$ is more important, and that it also leads to a correct fit to the experimental spectra in this energy range. It is therefore clearly demonstrated that the self-absorption phenomenon must necessarily be taken into account.

\section{B. Effect of $T_{e}$}

Figure 6 shows the effect of $T_{e}$ on $R_{i}(\hbar \omega)$ for indirect recombination (Si junction case) and fixed $x_{j} \sim 0.8 \mu \mathrm{m}$. We note that when $T_{e}$ increases, the EL intensity increases, and the emission peak shifts towards higher energies. In fact, as $T_{e}$ increases, the avalanche field applied increases and amplifies the impact ionization process. Consequently the population of hot carriers is amplified and these can reach high energy levels. As a result, the intensity of the EL increases and recombination of high energetic carriers involves high energetic photons, consistent with the shift of the peak position towards higher energy. The same behavior is obtained for direct recombination (not shown here) but, in that case, only a small variation of $T_{e}$ can induce a large variation of the EL intensity. Carriers recombining via direct process can not reach high energies because direct transitions are more

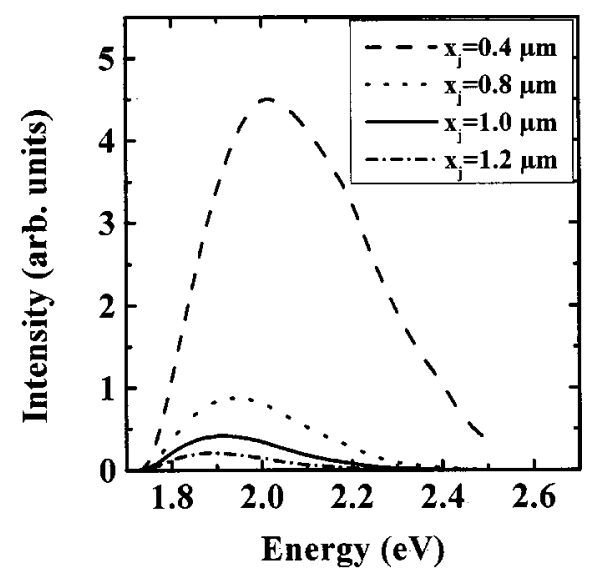

FIG. 5. Simulation of the indirect recombination peak intensity for different junctions depths for GaAs.

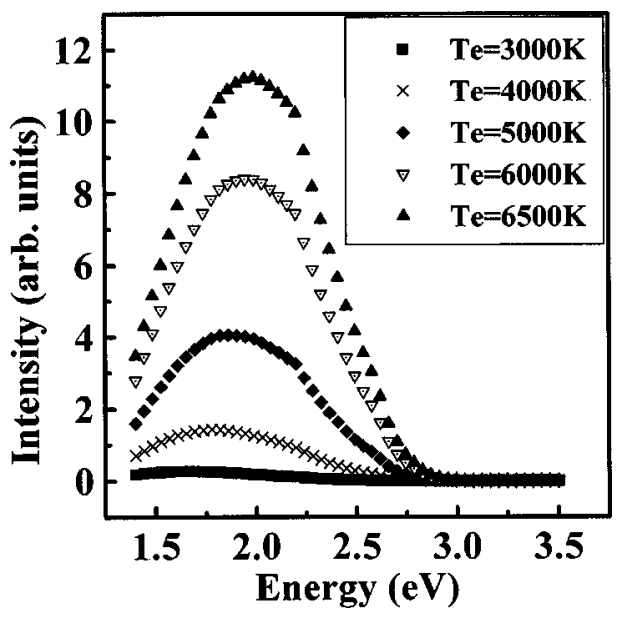

FIG. 6. Simulation of the electroluminescence intensity vs the energy for different carrier temperatures and for indirect recombination. Si with junction depth of $0.8 \mu \mathrm{m}$.

probable and consequently the carriers do not have enough time to reach higher values of $T_{e}$; whenever they do reach higher energies, they move towards indirect valleys and contribute to indirect transition emission. ${ }^{28}$

\section{Simulation of the experimental spectra}

The calculated spectra are obtained from two successive steps. The first step consists of the determination of $x_{j}$ : the value of $T_{e}$ is fixed and the shape of the spectrum in the high energy range is obtained from each value of $x_{j}$. In the second step, since $x_{j}$ is already fixed, $T_{e}$ is adjusted to fit the emission peak position.

\section{Si spectra}

Figure 7 shows a comparison of calculated and measured spectra of the $\mathrm{Si} p-n$ junction. Good agreement between the two spectra is achieved for $T_{e}=6500 \mathrm{~K}$ and $x_{j}=0.8 \mu \mathrm{m}$. The value of $x_{j}$ obtained here is in good accord with $0.8 \mu \mathrm{m}$, which was given by the supplier. The value of $T_{e}=6500 \mathrm{~K}$ is within the range of previously published values ${ }^{15,16}$ which were determined by different methods. In fact, Obeidat

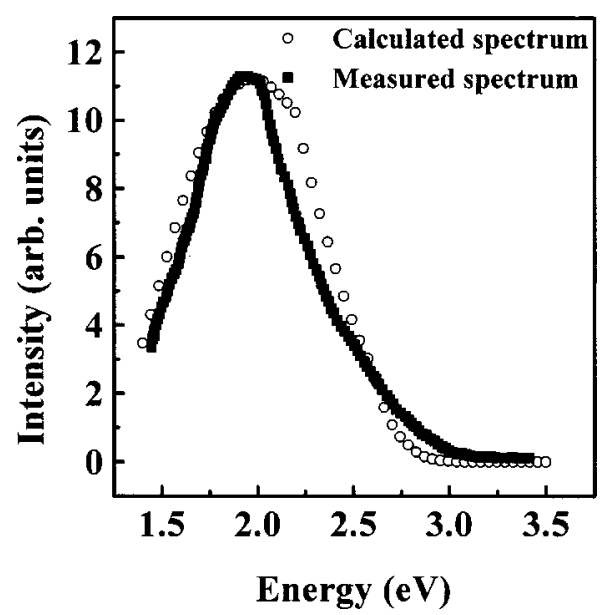

FIG. 7. Measured and simulated electroluminescence spectra vs the energy with self-absorption for $T_{e}=6500 \mathrm{~K}$ and $0.8 \mu \mathrm{m}$ junction depth for Si. 


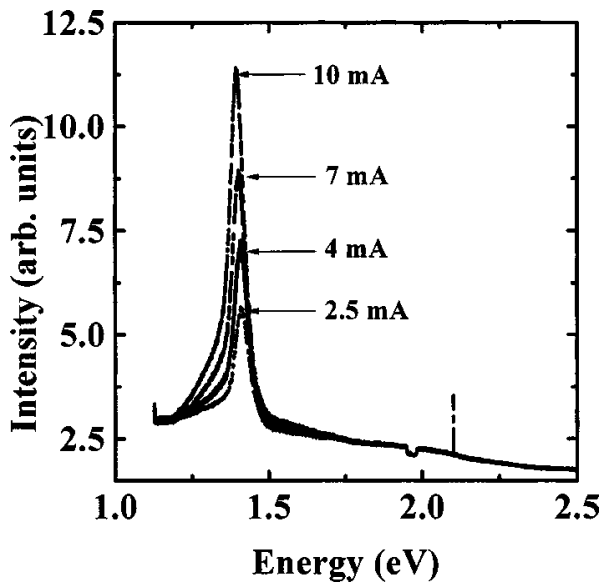

FIG. 8. Electroluminescence spectrum of the custom-fabricated GaAs $p-n$ junction during controlled avalanche breakdown for different values of reverse current. Correction of the detector's spectral response is included in all spectra.

et al. ${ }^{15}$ using a hot-carrier recombination model, have obtained a value of $7500 \mathrm{~K}$ for $\mathrm{Si} p-n$ junctions reverse biased at $6 \mathrm{~V}$ for current intensity of $10 \mathrm{~mA}$. Akil et al. ${ }^{16}$ using the same junctions, have estimated the hot-carrier temperature as between 3500 and $3800 \mathrm{~K}$ using a multimechanism model.

\section{GaAs spectra}

A slightly modified analysis is used in this case. The observed emission intensity at about $1.4 \mathrm{eV}$ varies significantly according to the current intensity applied (Fig. 8) which is a function of the magnitude of the electric field applied. For the indirect recombination peak, the effect of current applied on the measured light intensity seems to be nonexistent. This is probably due both to a small variation of the emitted light intensity induced by limited variation of the reverse current and by the high absorption coefficient in this energy range.

The amplitudes and positions of emission intensity peaks further depend on the hot-carrier temperature. The direct recombination peak position corresponds to the direct band gap energy of GaAs. The smaller peak is positioned $0.17 \mathrm{eV}$ above the indirect band gap energy of GaAs, indicating that carriers recombining via indirect process are more energetic. By comparison, silicon has an indirect gap of approximately $1.12 \mathrm{eV}$ and shows an indirect recombination peak localized near $1.8 \mathrm{eV}$. It is then necessary to use two electron temperatures $T_{e-d}$ and $T_{e \text {-ind }}$ to differentiate direct from indirect transitions. ${ }^{28}$ Figure 9 shows a comparison of measured and simulated indirect recombination EL spectra. Good agreement is obtained for carrier temperature $T_{e \text {-ind }}=3000 \mathrm{~K}$ and junction depth of $x_{j}=0.9 \mu \mathrm{m}$. Figure 10 shows a similar comparison near $1.4 \mathrm{eV}$, a direct recombination peak, with $x_{j}$ held at $0.9 \mu \mathrm{m}$ and $T_{e-d}=800 \mathrm{~K}$.

The value of $x_{j}$ is determined by matching the indirect recombination peaks because this high energy portion of the spectra is more strongly affected by self-absorption due to the high values of the absorption coefficients in this energy range. The value extracted agrees with the manufacturer's data to within the accuracy of the manufacturer's specifica-

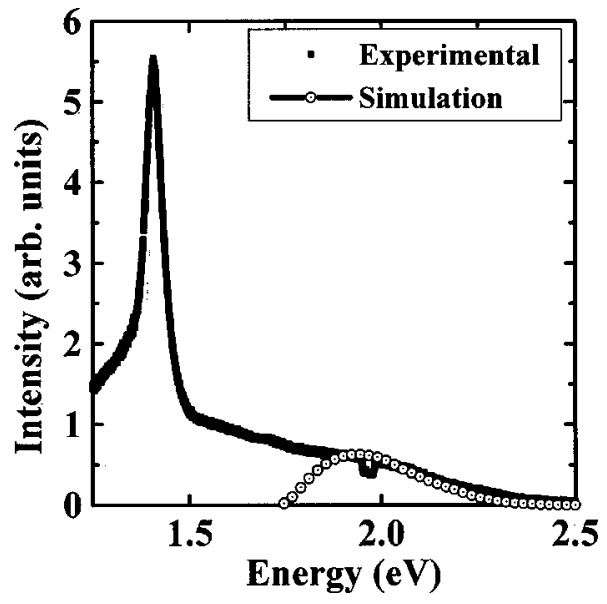

FIG. 9. Measured spectrum and simulated indirect recombination electroluminescence spectrum of $T_{e \text {-ind }}=3000 \mathrm{~K}$ and $x_{j}=0.9 \mu \mathrm{m}$ for GaAs.

tions. This determination of the junction depth by matching simulated and measured spectra can be used as a nondestructive technique to estimate the junction depth in GaAs, but can also be used for $\mathrm{Si}$ and other semiconductors.

The relatively low carrier temperature obtained for direct recombination is consistent with the fact that this is the most probable recombination process in GaAs. Since direct recombination carriers have shorter mean lifetimes, the carriers gain less energy in the applied field region. The relatively much lower probability for indirect recombination is associated with the longer lifetime of carriers participating in these events and with the greater energy gained from the junction field prior to recombination.

\section{Simulation of neutron irradiation effect}

Figure 11 shows measured EL spectra at controlled avalanche breakdown ${ }^{26}$ before and after $30 \mathrm{MeV}$ fast neutron irradiation for $12 \mathrm{~h}$ with fluence of $1.2 \times 10^{11} \mathrm{n} / \mathrm{cm}^{2}$ on a gate channel junction of a commercial silicon JFET (2N4416) fabricated by Siliconix. The additional peaks result from interference in the oxide layer overlaying the emitting junction. Similar results have already been obtained by Obeidat

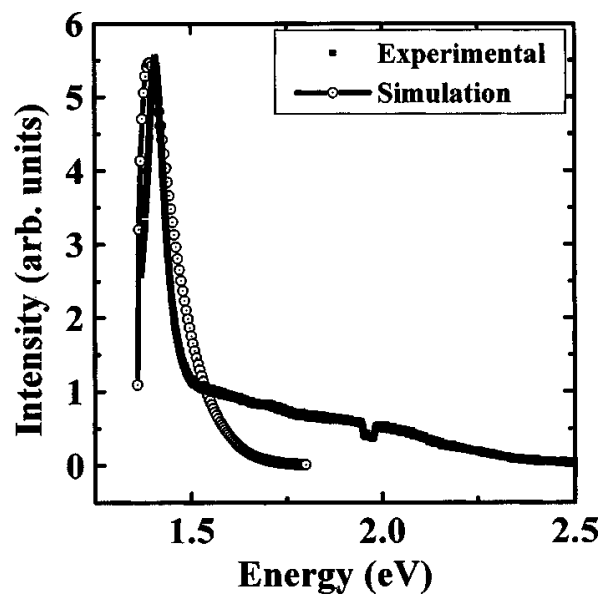

FIG. 10. Measured spectrum and simulated direct recombination electroluminescence spectrum for $T_{e-d}=800 \mathrm{~K}$ and $x_{j}=0.9 \mu \mathrm{m}$ for GaAs. 


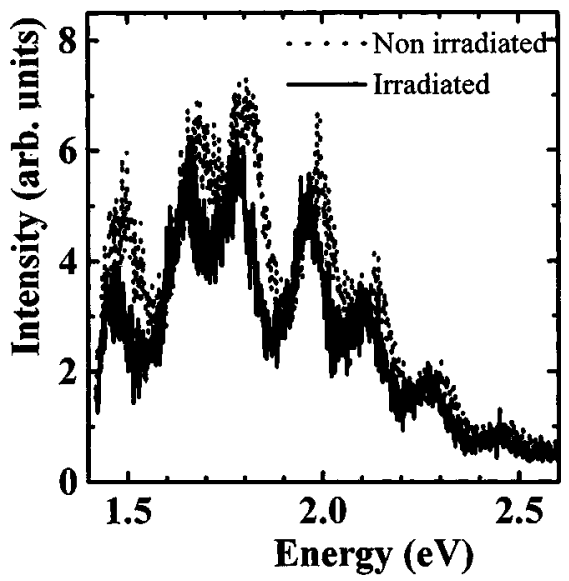

FIG. 11. Electroluminescence spectra of a gate-channel junction of a commercial Si JFET before and after $30 \mathrm{MeV}$ neutron irradiation for $12 \mathrm{~h}$ at controlled avalanche breakdown.

et $a l .{ }^{15}$ for bulk Si metal-oxide-semiconductor (MOS) transistors, and more recently by Karsenty et al. ${ }^{32}$ for silicon-oninsulator MOS transistors. It should be noted that the EL intensity decreases for all peaks and that the peak positions shift slightly towards lower energies after irradiation. These authors have attributed this behavior to densification of the oxide layer, that is, the refractive index changes from 1.46 to 1.48. By including interference of the light emitted within the passivation layer and applying the above emission model, for indirect recombination, we have simulated this spectrum for different values of the oxide refractive index. The results obtained are shown in Fig. 12, in which it is clear that the peaks positions shift slightly towards lower energies, but their respective intensities are still practically unchanged. However the refractive index increase is not sufficient to reproduce all the experimental changes observed.

To explain the observed decrease of the EL intensity, the effect of neutron irradiation on the SC must also be taken into account. In fact, the neutrons used here are so energetic (30 MeV) that they go through the SC, after passing through the oxide layer, and induce some displacement defects. ${ }^{33}$ The presence of these defects and the high electric field inside the junction result in both a reduction in free carrier density and a reduction of their mobility due to increased scattering of

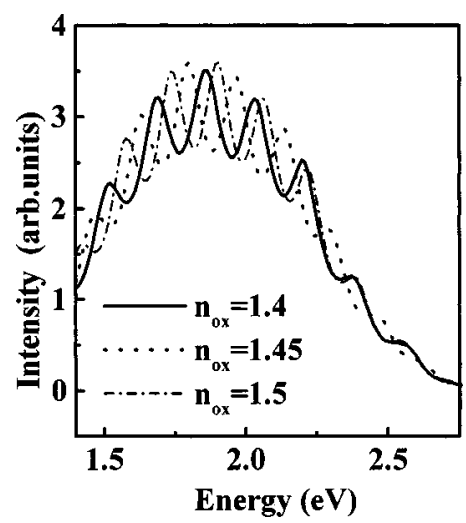

FIG. 12. Simulated electroluminescence spectra for different values of the refractive index $\left(n_{\mathrm{ox}}\right)$ of the oxide layer.

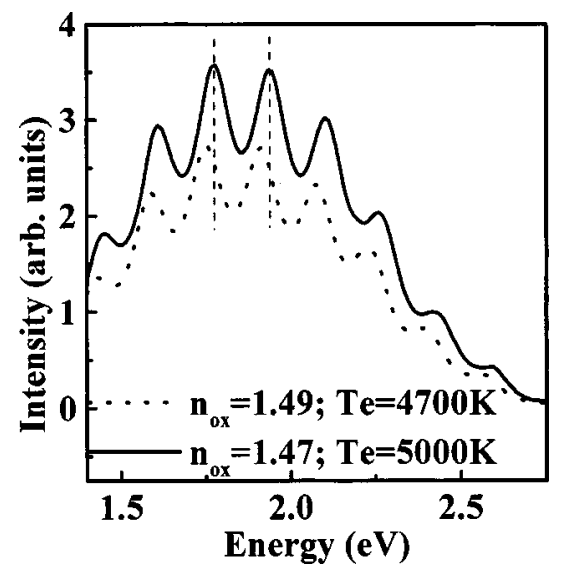

FIG. 13. Simulated electroluminescence spectrum that takes into account both densification of the oxide layer and the decrease in carrier temperature.

the remaining free carriers with the additional charge centers. ${ }^{34}$ Under avalanche breakdown, the free carrier density generated by impact ionization process is so great that the density of the trapped carriers can be neglected. Therefore, we can assume that interaction between hot carriers and additional charge centers results mainly in the braking of hot carriers, that is to say, their mobility decreases. Consequently, their energy and the corresponding temperature $T_{e}$ are reduced. ${ }^{35}$ The effect of neutron irradiation on the SC can then be simulated by a possible reduction in hot-carrier temperature. In fact, a decrease of $T_{e}$ results in a drop of the EL intensity (see Fig. 6).

Figure 13 shows simulated spectra taking into account both an increase of the refractive index of the oxide layer and a decrease of the hot-carrier temperature. The curves reproduce the same behavior as that observed experimentally. The good match between theoretical and experimental results confirms results of our analysis that both the SC and the overlaying oxide layer are affected by energetic neutron irradiation like the one used here.

\section{CONCLUSION}

In this work we presented a general model for the interpretation of EL spectra measured under avalanche breakdown conditions. It is an extension of the model of Yamada and Kiato $^{25}$ that took self-absorption into account.

The model presented is based on basic physical mechanisms such as interband (direct and indirect) transitions and self-absorption. It was validated by analysis of EL spectra obtained separately on indirect band gap semiconductors, such as Si junctions, and on direct band gap semiconductors, such as GaAs junctions. The model can be used as a noninvasive technique for the determination of the junction depth and is applicable to all types of SC $p-n$ junctions.

Furthermore, the model was successfully used to explain the observed effect of fast neutron irradiation on the EL spectra of Si $p-n$ junctions. Our work shows that analysis of a reverse biased $p-n$ junction EL spectrum is a convenient and useful tool for electronic device characterization and reliability studies. 


\section{ACKNOWLEDGMENTS}

This work was financially supported by both the Franco-Marocain Committee within the framework of MA/01/14 and the Moroccan government by PROgramme Thématique d'Appui a la Recherche Scientifique III D12/16. The authors would like to thank Isabelle Salesse (Centre d'Electronique et de Micro-optoelectronique de Montpellier , CNRS, Montpellier II University) for supplying the GaAs junctions.

${ }^{1}$ L. Selmi, M. Mastrapaqua, D. Boulin, J. D. Bude, M. Pavesi, E. Sangiorgi, and M. R. Pinto, IEEE Trans. Electron Devices 45, 802 (1998).

${ }^{2}$ H. Niwa, Y. Ohno, S. Kishimoto, K. Maezawa, T. Mizutani, Yamazaki, and T. Taniguchi, Jpn. J. Appl. Phys., Part 1 38, 1363 (1999).

${ }^{3}$ C. Domoto, N. Ohtani, K. Kuroyanaghi, P. O. Vaccaro, and N. Egami, Jpn. J. Appl. Phys., Part 1 38, 2577 (1999).

${ }^{4}$ G. Meneghesco, T. Grave, M. Manfredi, M. Pavesi, C. Canali, and E. Zanoni, IEEE Trans. Electron Devices 47, 2 (2000).

${ }^{5}$ N. Cavassilas, F. Aniel, P. Boucaud, R. Adde, H. Maher, J. Décobert, and A. Scavennec, J. Appl. Phys. 87, 2548 (2000).

${ }^{6}$ N. Shigekawa, K. Shiojima, and T. Sumitsu, J. Appl. Phys. 92, 531 (2002).

${ }^{7}$ K. Onodera, K. Nishimura, and T. Furuta, IEEE Trans. Electron Devices 46, 2170 (1999).

${ }^{8}$ R. Gaddi, G. Meneghesco, M. Pavesi, M. Peroni, C. Canali, and E. Zanoni, IEEE Trans. Electron Devices 20, 372 (1999).

${ }^{9}$ H. Sazaki, M. Abe, T. Fujioka, K. Hayashi, K. Mizuguchi, B. Yea, T. Osaki, K. Sugahara, and R. Konishi, Jpn. J. Appl. Phys., Part 1 37, 455 (1998).

${ }^{10}$ H. Sazaki, K. Hayashi, T. Fujioka, K. Mizuguchi, B. Yea, T. Osaki, K. Sugahara, and R. Konishi, Jpn. J. Appl. Phys., Part 1 37, 4301 (1998).

${ }^{11}$ R. Newman, Phys. Rev. 100, 700 (1955).

${ }^{12}$ A. G. Chynoweth and K. G. Mckay, Phys. Rev. 102, 369 (1956).

${ }^{13}$ M. Herzog and F. Koch, Appl. Phys. Lett. 53, 2620 (1988).

${ }^{14}$ J. Kramer, P. Seitz, E. F. Steigmeier, H. Auderest, B. Delley, and H. Baltes, Sens. Actuators A 37-38, 527 (1993).

${ }^{15}$ A. T. Obeidat, Z. Kalayjian, A. G. Andreou, and J. B. Khurgin, Appl. Phys. Lett. 70, 470 (1997)
${ }^{16}$ N. Akil, S. E. Kerns, D. V. Kerns, Jr., A. Hoffmann, and J. P. Charles, IEEE Trans. Electron Devices 46, 1022 (1999).

${ }^{17}$ C. W. Liu, S. T. Chang, W. T. Liu, M.-J. Chen, and C.-F. Lin, Appl. Phys. Lett. 77, 4347 (2000).

${ }^{18}$ N. Akil, V. E. Houtsma, P. LeMinh, J. Holleman, V. Zieren, D. de Mooij, P. H. Woerlee, A. van den Berg, and H. Wallinga, J. Appl. Phys. 88, 1916 (2000)

${ }^{19}$ A. L. Lacaita, F. Zappa, S. Bigliardi, and M. Manfredi, IEEE Trans. Electron Devices 40, 577 (1993).

${ }^{20}$ W. Haecker, Phys. Status Solidi A 25, 301 (1974).

${ }^{21}$ J. Schewchun and L. Y. Wei, Solid-State Electron. 8, 485 (1965).

${ }^{22}$ P. A. Wolff, J. Phys. Chem. Solids 16, 184 (1960).

${ }^{23}$ J. Bude, N. Sano, and A. Yoshii, Phys. Rev. B 45, 5848 (1992).

${ }^{24}$ D. K. Gautam, W. S. Khokle, and K. B. Garg, Solid-State Electron. 31, 219 (1998).

${ }^{25}$ S. Yamada and M. Kiato, Jpn. J. Appl. Phys., Part 1 32, 4555 (1993).

${ }^{26}$ S. Forster, A. Hoffmann, J.-P. Charles, S. E. Kerns, D. V. Kerns, Jr., M. de la Bardonie, and P. Mialhe, IEEE Proceedings of 5th European Conference on Radiation and its Effects on Components and Systems Reliability of Electron Devices, Fontevraud France, 1999, pp. 508-511.

${ }^{27}$ M. Lahbabi, A. Ahaitouf, E. Abarkan, M. Fliyou, A. Hoffmann, J. P. Charles, B. L. Bhuva, S. E. Kerns, and D. V. Kerns, Jr., Appl. Phys. Lett. 77, 3182 (2000)

${ }^{28}$ M. Lahbabi, A. Ahaitouf, E. Abarkan, M. Fliyou, A. Hoffmann, J. P. Charles, B. L. Bhuva, S. E. Kerns, and D. V. Kerns, Jr., Appl. Phys. Lett. 80, 1004 (2002)

${ }^{29}$ S. M. Sze, Physics of Semiconductor Devices, 2nd ed. (Wiley, New York, 1981), p. 13

${ }^{30}$ P. A. Wolff, Phys. Rev. 95, 1415 (1954).

${ }^{31}$ D. E. Aspnes and A. A. Studna, Phys. Rev. B 27, 985 (1983).

${ }^{32}$ A. Karsenty, A. Sa'ar, N. Ben-Yosef, and J. Shappir, Appl. Phys. Lett. 82, 4830 (2003)

${ }^{33}$ J. R. Srour and J. M. McGarrity, Proc. IEEE 76, 1443 (1988).

${ }^{34}$ F. B. Mc Lean, J. M. Mc Garritu, C. J. Scozzie, C. W. Tipton, and W. M. Delancey, IEEE Trans. Nucl. Sci. 41, 1884 (1994).

${ }^{35}$ A. Ahaitouf, M. Lahbabi, M. Fliyou, E. Abarkan, A. Hoffmann, and J.-P. Charles, ICM '2001 Proceedings, 13th International Conference on Microelectronics, Rabat, Morocco, 2001, pp. 71-74. 